\title{
Rosuvastatin Use Implicated in the Drug Reaction with Eosinophilia and Systemic Symptoms
}

\author{
Syed Farrukh Mustafa ${ }^{1}$, Meer R. Zafar ${ }^{2,3}$, Timothy W. Miller ${ }^{3}$ \\ 1. Internal Medicine, William Beaumont Hospital, Royal Oak, USA 2. Internal Medicine, Sisters of Charity Hospital, \\ Buffalo, USA 3. Internal Medicine, Jacobs School of Medicine and Biomedical Sciences, Buffalo, USA
}

Corresponding author: Meer R. Zafar, meer_rabeel@hotmail.com

\begin{abstract}
Drug reaction with eosinophilia and systemic symptoms (DRESS) is a life-threatening drug-induced hypersensitivity reaction that is most closely associated with antiepileptics and antibiotics. While cases of DRESS are rare, here we present a case of DRESS in an adult male following administration of rosuvastatin who presented with fevers, generalized rash, and facial fullness. Vitals on presentation were temperature $102^{\circ} \mathrm{F}$, pulse $95 / \mathrm{min}$, blood pressure $95 / 47 \mathrm{mmHg}$, and respiratory rate of $14 / \mathrm{min}$. His physical examination revealed scleral icterus, generalized blanching maculopapular rash, facial fullness, and right upper quadrant tenderness. Laboratory investigations found hemoglobin $10 \mathrm{gm} / \mathrm{dl}$, white blood cell count $16.0 \mathrm{~K} / \mathrm{uL}$, peripheral eosinophil count 1,700 K/uL, alkaline phosphatase 2,501 U/L, aspartate transaminase $620 \mathrm{U} / \mathrm{L}$, alanine transaminase $680 \mathrm{U} / \mathrm{L}$, total bilirubin $13.2 \mathrm{mg} / \mathrm{dl}$ with a direct component of $9 \mathrm{mg} / \mathrm{dl}$, blood urea nitrogen $66 \mathrm{mg} / \mathrm{dl}$, creatinine $5.20 \mathrm{mg} / \mathrm{dl}$, glomerular filtration rate $8 \mathrm{ml} / \mathrm{min}$, and immunoglobulin $\mathrm{E}$ level $623 \mathrm{IU} / \mathrm{mL}$. Serology for viral hepatitis, Epstein-Barr virus, cytomegalovirus, and human herpesvirus 6 was negative. Computed tomographic scan of chest, abdomen, and pelvis showed generalized lymphadenopathy. Over the next week, the patient deteriorated clinically with worsening transaminitis and oliguric acute renal failure requiring renal replacement therapy. As per the European Registry of Severe Cutaneous Adverse Reaction Criteria (RegiSCAR), the probability of rosuvastatin-induced DRESS syndrome was scored as "definite." He was treated with systemic and topical glucocorticoids leading to a gradual improvement in his symptoms. Skin biopsy was suggestive of DRESS syndrome as well. Since DRESS carries such a significant risk of mortality between $10 \%$ and $20 \%$, DRESS must be recognized and treated as soon as symptoms present. Clinicians should also be aware that statins, one of the most commonly prescribed drugs, are also a potential cause DRESS.
\end{abstract}

Received 01/30/2020 Review began 02/15/2020 Review ended 02/22/2020 Published 02/25/2020

\section{(c) Copyright 2020}

Mustafa et al. This is an open access article distributed under the terms of the Creative Commons Attribution License CC-BY 4.0., which permits unrestricted use, distribution, and reproduction in any medium, provided the original author and source are credited.
Categories: Internal Medicine, Allergy/Immunology, Hematology

Keywords: drug reaction, eosinophilia, rosuvastatin, generalized rash, generalized lymphadenopathy, glucocorticoids

\section{Introduction}

Drug reaction with eosinophilia and systemic symptoms (DRESS) is a severe, potentially life-threatening drug-induced reaction characterized by fever, skin eruption, hematologic abnormalities, and multi-organ dysfunction [1]. The skin rash can range from a pruritic maculopapular eruption that progresses to a confluent erythematous rash with follicular accentuation, frequently involving more than $50 \%$ of the body surface area [2]. Hematologic abnormalities in DRESS include elevated leukocyte count with significant eosinophilia and atypical lymphocytosis [1]. Multiple internal organs can be involved with liver dysfunction being the most common, ranging from transient mild elevation in liver function tests in most cases to fulminant liver failure [3]. Other common manifestations include lung involvement (pneumonitis, pleural effusions) and kidney involvement (interstitial nephritis). According to a study, the estimated annual incidence of DRESS is 0.9/100,000 [4]. It is associated with anywhere between 1/1000 and 1/10,000 drug exposures and mortality is $10 \%$ to $20 \%$ [5]. Dibek Misirlioglu et al. reported that antibiotics were the most common (50\%) medication in the etiology; $87.5 \%$ of the suspected antibiotics were beta-lactams and $12.5 \%$ were macrolides. Antiepileptics were the second (37.5\%) most common class of drugs in the etiology [6]. Statin use has not been commonly associated with DRESS. We describe a case of DRESS in a patient with exposure to rosuvastatin.

\section{Case Presentation}

A 54-year-old male with a past medical history of dyslipidemia and asthma presented with acute onset of fever, facial fullness, and a generalized rash of two-day duration. He had a recent travel history to Europe (Greece and Turkey), but had no exposure to insects, animals or tick bites. His home medications include rosuvastatin $10 \mathrm{mg}$ daily. His vitals were temperature $102^{\circ} \mathrm{F}$, pulse $95 / \mathrm{min}$, blood pressure $95 / 47 \mathrm{mmHg}$, and respiratory rate $14 / \mathrm{min}$. On physical examination, the patient had scleral icterus, generalized blanching maculopapular rash involving $>50 \%$ of body surface area, facial fullness, and right upper quadrant tenderness. Laboratory investigations revealed hemoglobin $10 \mathrm{gm} / \mathrm{dl}$, white blood cell count $16.0 \mathrm{~K} / \mathrm{uL}$, peripheral eosinophil count 1,700 K/uL, alkaline phosphatase 2,501 U/L, aspartate transaminase 620 U/L, alanine transaminase $680 \mathrm{U} / \mathrm{L}$, total bilirubin $13.2 \mathrm{mg} / \mathrm{dl}$ with a direct component of $9 \mathrm{mg} / \mathrm{dl}$, blood urea 


\section{Cureus}

nitrogen $66 \mathrm{mg} / \mathrm{dl}$, creatinine $5.20 \mathrm{mg} / \mathrm{dl}$, glomerular filtration rate $8 \mathrm{ml} / \mathrm{min}$, and immunoglobulin E (IgE) level $623 \mathrm{IU} / \mathrm{mL}$. Serology for viral hepatitis, Epstein-Barr virus, cytomegalovirus, and human herpesvirus 6 was negative. The patient was started on intravenous normal saline, vancomycin, and piperacillintazobactam as he met systemic inflammatory response syndrome criteria, but symptoms did not improve. A computed tomographic scan of the chest, abdomen, and pelvis showed generalized lymphadenopathy (Figure 1). Over the next few days, he deteriorated clinically with worsening renal failure requiring renal replacement therapy. Skin biopsy was performed, antibiotics were discontinued, and the patient was started on intravenous methylprednisolone $120 \mathrm{mg} /$ day and topical clobetasol propionate $0.05 \%$ ointment with rapid improvement over several days and was discharged on oral prednisone. Biopsy of skin showed vacuolar and interface dermatitis with perivascular lymphocytic and eosinophilic infiltrate (Figure 2). These findings were consistent with DRESS syndrome. At one-month follow-up, the patient was asymptomatic.

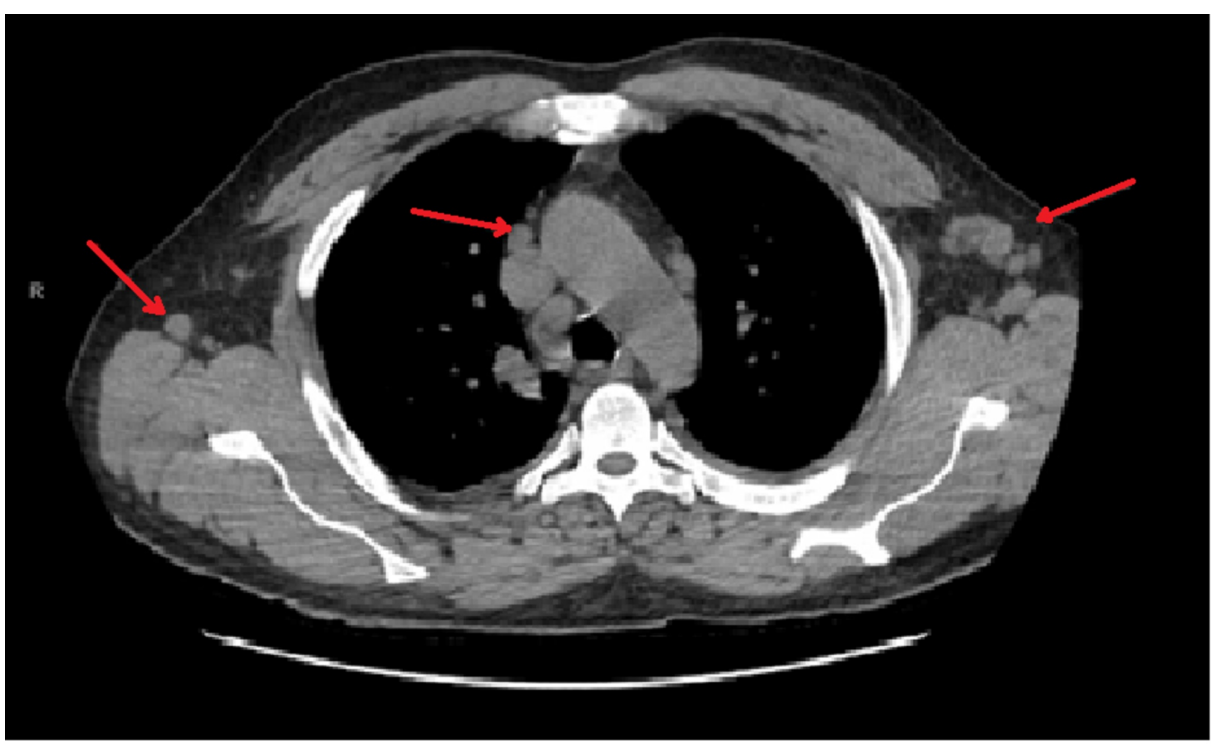

FIGURE 1: CT scan of the chest showing enlarged mediastinal and axillary lymph nodes.

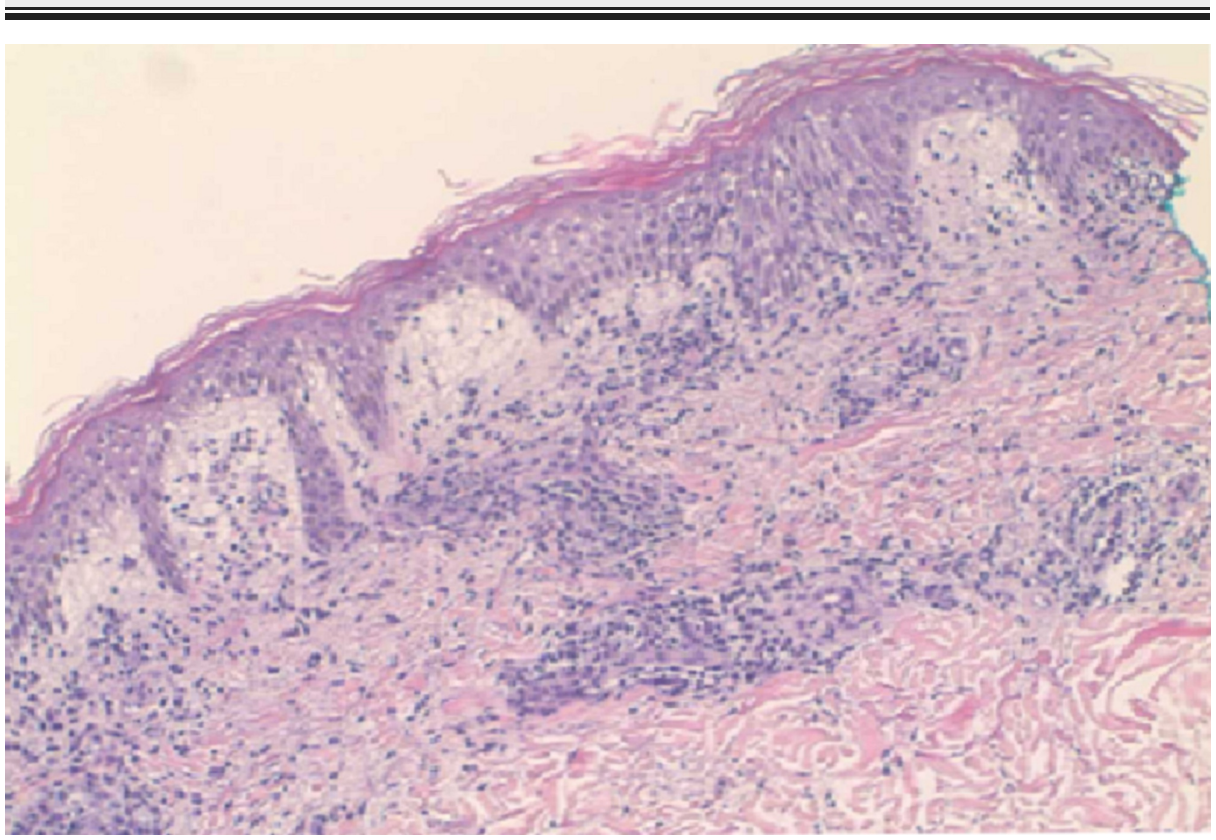

FIGURE 2: Punch biopsy of the skin in anterior forearm (10x).

\section{Discussion}

We present a case of DRESS syndrome in a patient with rosuvastatin use. As the onset of fever and rash correlated with recent travel to Europe, infectious etiology was assumed obscuring the correct diagnosis and 


\section{Cureus}

our initial diagnostic approach. Malignancy and vasculitis became prominent differentials with the emerging evidence of multi-organ dysfunction (liver and kidneys), clinical deterioration, and generalized lymphadenopathy on computer tomography imaging; however, increased eosinophil count and elevated IgE levels were more consistent with DRESS syndrome.

The onset of symptoms in DRESS is usually delayed, ranging from two to six weeks after initiation of the drug [7]. Our patient reported that he was prescribed rosuvastatin a few years ago by his primary physician, but he never took it consistently. He was non-compliant with his rosuvastatin for about one year before starting it again about six weeks before the presentation. One possible explanation could be that inconsistent use of the drug causes initial sensitization and re-exposure causes a severe reaction manifesting as DRESS. It has been shown that following re-exposure, the symptoms may occur more rapidly and maybe more severe [8]. Our patient did not recall having a reaction to rosuvastatin when he first started taking it. He may have had a mild subclinical reaction at that time or the inconsistent use may not have been enough to sensitize his immune system to mount a generalized hypersensitivity reaction as seen in DRESS. According to our literature review, only one case of DRESS associated with atorvastatin use has been reported [9].

RegiSCAR (European Registry of Severe Cutaneous Adverse Reactions) is a scoring system developed to aid in the diagnosis of DRESS (Table 1). It takes into account various clinical features and laboratory investigations and depending on the score, cases of DRESS are grouped into four categories: no case, possible case, probable case, and definite case [2]. In our patient, the calculated RegiSCAR score was $>6$, indicating a "definite" case of DRESS. The limitation to RegiSCAR criteria is that some of the parameters cannot be assessed until later in the disease course (e.g., skin biopsy findings and disease duration). Hence, it is more useful for retrospectively diagnosing but still serves as a useful tool to guide the collection of clinical data.

\begin{tabular}{|c|c|c|}
\hline Item & Present & Absent \\
\hline Fever $>38.5^{\circ} \mathrm{C}$ & 0 & -1 \\
\hline Enlarged lymph node ( >1 cm at least two sites) & 1 & 0 \\
\hline Eosinophilia: $>700$ cells $/ \mu \mathrm{L}$ or $>10 \%$ & 1 & 0 \\
\hline Eosinophilia: >1500 cells/ $\mu \mathrm{L}$ or >20\% & 2 & 0 \\
\hline Rash $>50 \%$ body surface area & 1 & 0 \\
\hline >2 of facial edema, purpura, desquamation, or infiltration & 1 & 0 \\
\hline Skin biopsy suggesting alternate diagnosis & -1 & 0 \\
\hline One organ involvement & 1 & 0 \\
\hline Two or more organ involvement & 2 & 0 \\
\hline Disease duration $>15$ days & 0 & -2 \\
\hline Investigation for alternative cause negative & 1 & 0 \\
\hline
\end{tabular}

TABLE 1: RegiSCAR scoring system for DRESS. (16) Total score <2: excluded; 2-3: possible; 4-5: probable; >6: definite.

DRESS, drug reaction with eosinophilia and systemic symptoms; RegiSCAR, European Registry of Severe Cutaneous Adverse Reaction.

\section{Conclusions}

Given that statins are not commonly implicated in DRESS syndrome, and the patient's initial clinical picture seemed to be related to an infectious etiology, there was some delay in diagnosis. Once correctly diagnosed, the patient improved with systemic glucocorticoids and supportive treatment. DRESS syndrome can be associated with significant morbidity and mortality, and hence should be always considered in the differential of cutaneous eruptions with systemic signs and symptoms. It may occur in the setting of drugs not classically known to be associated with it. Hence, a thorough reconciliation of patient's medication should be performed, and particularly recent medication changes should be explored. The culprit drug should be immediately discontinued, and the reaction should be documented in the health record as a severe allergy to avoid re-exposure. Clinicians should be aware that statins, which are one of the commonest prescription drugs, can potentially be associated with DRESS syndrome. 


\section{Additional Information}

\section{Disclosures}

Human subjects: Consent was obtained by all participants in this study. Conflicts of interest: In compliance with the ICMJE uniform disclosure form, all authors declare the following: Payment/services info: All authors have declared that no financial support was received from any organization for the submitted work. Financial relationships: All authors have declared that they have no financial relationships at present or within the previous three years with any organizations that might have an interest in the submitted work. Other relationships: All authors have declared that there are no other relationships or activities that could appear to have influenced the submitted work.

\section{References}

1. Cacoub P, Musette P, Descamps V, et al.: The dress syndrome: a literature review . Am J Med. 2011, 124:588597. 10.1016/j.amjmed.2011.01.017

2. Kardaun SH, Sekula P, Valeyrie-Allanore L, et al.: Drug reaction with eosinophilia and systemic symptoms (DRESS): an original multisystem adverse drug reaction. Results from the prospective RegiSCAR study. Br J Dermatol. 2013, 169:1071-1080. 10.1111/bjd.12501

3. Björnsson E, Olsson R: Outcome and prognostic markers in severe drug-induced liver disease . Hepatology. 2005, 42:481-489. 10.1002/hep.20800

4. Muller P, Dubreil P, Mahé A, et al.: Drug hypersensitivity syndrome in a West-Indian population . Eur J Dermatol. 2003, 13:478-481.

5. Lopez-Rocha E, Blancas L, Rodriguez-Mireles K, et al.: Prevalence of DRESS syndrome. Rev Alerg Mex. 2014, 61:14-23.

6. Dibek Misirlioglu E, Guvenir H, Bahceci S, et al.: Severe cutaneous adverse drug reactions in pediatric patients: a multicenter study . J Allergy Clin Immunol Pract. 2017, 5:757-763. 10.1016/j.jaip.2017.02.013

7. Tas S, Simonart T: Management of drug rash with eosinophilia and systemic symptoms (DRESS syndrome): an update . Dermatology. 2003, 206:353-356.

8. Bocquet H, Bagot M, Roujeau JC: Drug-induced pseudolymphoma and drug hypersensitivity syndrome (drug rash with eosinophilia and systemic symptoms: DRESS). Semin Cutan Med Surg. 1996, 15:250-257. 10.1016/s1085-5629(96)80038-1

9. Gressier L, Pruvost-Balland C, Dubertret L, Viguier M: Atorvastatin-induced drug reaction with eosinophilia and systemic symptoms (DRESS). Ann Dermatol Venereol. 2009, 136:50-53. 10.1016/j.annder.2008.07.063 